\title{
INDICADORES DA MODERNIZAÇÃO AGRÍCOLA DO ESTADO DE MATO GROSSO
}

\author{
Elizangela Beckmann ${ }^{1}$ \\ Antônio Cordeiro de Santana ${ }^{2}$
}

\section{RESUMO}

A modernização da produção agrícola de Mato Grosso resultou das combinações entre insumos químicos, máquinas e equipamentos mecânicos e biotecnologia com as variáveis relacionadas à educação, assistência técnica, inovações na organização e gestão da atividade. Assim, para destacar o estado na produção agrícola foram identificados e analisados os fatores que determinam a modernização da agricultura e apresentada sua configuração nos municípios por meio de um Índice de Modernização Agrícola Municipal. Para tanto, utilizou-se a Análise Fatorial para extrair os fatores e hierarquizar os municípios de acordo com o nível de modernização. Foram identificados cinco fatores responsáveis pela modernização agrícola de Mato Grosso e, dentre estes, a agricultura de altos insumos se mostrou como um fator positivo na maioria dos municípios, enquanto o fator produtivo se mostrou negativo na maior parte dos municípios do estado. O nível de modernização apresentado pela maioria dos municípios foi baixo, sendo que nenhum obteve índice alto, o que reafirma o caráter ainda extensivo da agricultura matogrossense e a distribuição não homogênea da tecnologia. Os resultados obtidos neste estudo auxiliam visualizar os pontos fortes e fracos relacionados a modernização da agricultura em Mato Grosso, permitindo ações condizentes com o contexto atual de cada município.

Palavras-chave: agricultura, análise fatorial, índice de modernização.

\section{INDICATORS OF AGRICULTURAL MODERNIZATION OF MATO GROSSO}

\begin{abstract}
The modernization of agricultural production in Mato Grosso resulted from combinations of chemical inputs, machinery and mechanical equipment and biotechnology with the variables related to education, technical assistance, innovation in organization and management of the activity. So to highlight the state in agricultural production were identified and analyzed the factors that determine the modernization of agriculture and presented its configuration in the municipalities
\end{abstract}

\footnotetext{
1 Graduada em Ciências Econômicas pela Universidade do estado de Mato Grosso. Mestre em Agronegócios e Desenvolvimento Regional pela Universidade Federal de Mato Grosso. Doutora em Ciências Agrárias pela Universidade Federal Rural da Amazônia. E-mail: elizangela.beckmann@hotmail.com.

2 Graduado em Agronomia pela Universidade Federal do Ceará. Mestre em Economia Rural pela Universidade Federal do Ceará. Doutor em Economia Aplicada pela Universidade Federal de Viçosa. Professor Titular da Universidade Federal Rural da Amazônia. E-mail: acsufra@gmail.com.
} 
through a Municipal Agricultural Modernization Index. For this, it was used the factor analysis to extract factors and rank municipalities according to the level of modernization. Have been identified five factors responsible for agricultural modernization of Mato Grosso and, among these, the high-input agriculture is shown as a positive factor in most municipalities, while the productive factor was negative in most municipalities. The level of modernization presented by most municipalities was low, and no obtained high index, which reaffirms the still extensive character of Mato Grosso agriculture and heterogeneous distribution of technology. The results of this study help visualize the strengths and weaknesses related to modernization of agriculture in Mato Grosso, allowing actions consistent with the current context of each municipality.

Keywords: Agriculture, Factorial Analysis, Modernization Index.

\section{INTRODUÇÃO}

O setor agropecuário brasileiro vem passando por intensivo processo de modernização por meio da utilização de novas tecnologias, combinando insumos químicos (fertilizantes, corretivos e defensivos químicos para controle de pragas, doenças e ervas daninhas) e máquinas e equipamentos modernos (tratores, colheitadeiras, grade, arado e implementos), cujo resultado aparece no incremento da produtividade do trabalho e da terra (CUNHA et al., 2008; RAMMINGER; GRASEL; ZAVALA, 2008; GASQUES; VIEIRA FILHO; NAVARRO, 2010). Inclui-se, ainda, na dinâmica desse processo de modernização, a educação, a assistência técnica, a biotecnologia em sementes mais resistentes e mais produtivas, além das inovações na organização e gestão da agropecuária.

O uso de máquinas e equipamentos contribuiu para o aumento da produção e, para tornar o sistema mais eficiente, foram incorporadas tecnologias de precisão e técnicas de cultivos mitigadoras de impacto sobre o meio ambiente. São os casos de máquinas para plantio, colheita e pulverização com GPS (Global Positioning System), do sistema Integração Lavoura Pecuária (ILP) e da biotecnologia em sementes (FERO, 2014).

Nesse contexto, a modernização agropecuária, a partir de 1960, beneficiada pelas políticas de crédito e de substituição de importações de insumos e equipamentos agrícolas, produziu a transformação da base técnica da produção e aumentou a produtividade. Portanto, a evolução da agropecuária fundamentou-se nas mudanças tecnológicas atreladas ao que se denominou de Revolução Verde (EHLERS, 1994; SEPULCRI; PAULA, 2005; NUNES, 2007). Assim, a mudança na base produtiva teve como finalidade a modernização de algumas áreas do campo brasileiro para atender a demanda mundial de alimentos e contribuir para gerar superávits na balança comercial (PESSÔA; MATOS, 2013). No II Plano Nacional de Desenvolvimento (II PND), os investimentos foram redirecionados e a relação entre agricultura e indústria melhorou. Com este plano ocorreram investimentos em infraestrutura rural, reorganização dos serviços de extensão e pesquisa agrícola e de crédito subsidiado para promover a industrialização da agricultura (SANTANA, 1994).

Com efeito, a modernização da agropecuária brasileira pode ser dividida em três fases. A primeira, 1960-1970, limita-se à transformação da base técnica pela incorporação de tratores e máquinas agrícolas e foi impulsionada por políticas governamentais voltadas para a agricultura. A segunda fase, 1970-1990, caracteriza-se pela industrialização da produção rural, com a implantação de 
indústrias de bens de produção e de alimentos. Na terceira, ou fase atual, a partir de 1990, ocorre a integração entre a agricultura e a indústria, além da incorporação da biotecnologia, que proporcionou benefícios à agropecuária, tais como: simplificação do manejo, redução de custos e ganhos de produtividade (SEPULCRI; PAULA, 2005; MAZZOLENI; OLIVEIRA, 2010).

Em decorrência da globalização e da presença de grandes agroindústrias e empresas do atacado e do varejo, o mercado agrícola mundial ganhou nova dinâmica a partir dos anos 1990 e foi capaz de intensificar as transformações tecnológicas no campo (NUNES, 2007). Assim, no século XXI, a modernização da agropecuária não pode mais ser vista somente sob a ótica do uso intensivo de máquinas e insumos modernos. Deve-se agregar o processo de modificações ocorridas nas relações sociais de produção, envolvendo melhorias em educação, pesquisa e gestão (TEIXEIRA, 2005).

Conforme estudo do Instituto de Pesquisa Econômica Aplicada (IPEA, 2010), o crescimento da produção e da produtividade resultou em uma ampliação das cadeias produtivas de base agrícola, com a expansão de vínculos com os elos da indústria de fornecimento e de processamento. Além disso, aumentou a relação com serviços sofisticados, de pesquisa, experimentação e difusão, além de consultorias em áreas da tecnologia da informação, genética, agricultura de precisão, e de todos os demais tipos de serviços relacionados à propriedade e às indústrias da cadeia de produção.

O conceito de modernização, de acordo com Balsan (2006), relaciona-se com o espaço e o tempo. Com o espaço porque distingue agricultores com graus diferentes de modernização, num mesmo lapso de tempo, e temporal porque um mesmo sistema de produção agrícola pode evoluir de tradicional à moderno no decorrer do tempo. Isto tende a ocorrer nos municípios de Mato Grosso, que se diferenciam por condições de relevo, solo e clima, fazendo com que uns sejam mais aptos à agricultura e consequentemente viabilizem a modernização da agricultura em relação a outros que não apresentam tais características.

As principais atividades econômicas do estado que contribuíram com a modernização da agropecuária, desde a década de 1980, são as culturas de arroz, soja, algodão, milho, cana-de-açúcar e a criação de bovinos, suínos e aves, além do plantio de florestas (PEREIRA; MENDES, 2002). Destas, destacam-se os cultivos de algodão, soja, milho e a criação de bovinos, tendo a produção de soja, entre 1980 e 2006, um incremento de mais de $100 \%$, assim como o algodão e o milho. Em 2006, o estado produziu 15.594.221 milhões de toneladas de soja, 13.552.228 milhões de toneladas cana-de-açúcar e 4.228.423 milhões de toneladas de milho (IBGE, 2016).

Estes incrementos na produção agrícola podem ser justificados pela expansão da fronteira agrícola em direção ao Centro-Oeste, iniciada na década de 1980 e, também, por ganhos em produtividade, proporcionados pelas inovações tecnológicas, biológicas e de organização e gestão que vêm sendo aderidas na agricultura. Em 2006 a produtividade da soja, cultivo mais expressivo em Mato Grosso, era de 2,68 toneladas por hectare, já em 2014 mesmo com aumento da área cultivada em $48 \%$, a produtividade passou para 3,07 toneladas por hectare (IBGE, 2016).

De maneira geral, vários estudos analisaram a modernização agropecuária no Brasil. Hoffmann (1992) determinou o grau de modernização agrícola de 157 microrregiões brasileiras homogêneas dos estados de Pernambuco, Bahia, São Paulo, Paraná, Santa Catarina, Rio Grande do Sul, Mato Grosso do Sul e Mato Grosso utilizando dados dos Censos Agropecuários de 1975 e 1980. Identificou dois fatores, um relacionado a intensidade de exploração da terra e outro a relação capital/trabalho. Quanto a Mato Grosso, concluiu que ambos os fatores ficaram 
negativos confirmando o fato de a agricultura ser extensiva no estado naquele período.

Souza e Lima (2003) estudaram a intensidade e a dinâmica da modernização agrícola no Brasil, concluindo que a modernização ocorre de maneira diferenciada entre quatro grupos de estados, sendo que o grupo que incluiu Mato Grosso foi considerado de modernização superior; Ferreira Jr., Baptista e Lima (2004) analisaram a modernização das microrregiões de Minas Gerais, encontrando três fatores, intensidade do uso da terra, relação capital/trabalho e área irrigada, que permitiram agrupar as microrregiões em 10 grupos de acordo com nível tecnológico. Campos, Pereira e Teixeira (2014) também trataram da modernização mineira para o período de 1996 e 2006, utilizando análise fatorial múltipla e agrupamentos, concluindo que os estabelecimentos têm baixa capacidade de absorver novas tecnologias e, que há uma relação inversa entre meio ambiente e tecnologia.

Pinto e Coronel (2015) determinaram os fatores de modernização para os municípios e mesorregiões do Rio Grande do Sul, calculando também o Índice de Modernização Agrícola, o qual teve resultado médio acima de 0,5. Costa et al. (2012) demonstraram os fatores e o nível de modernização dos estados brasileiros. O resultado indica que dos 27 estados brasileiros analisados, Mato Grosso está entre os mais modernos e foi o primeiro colocado no fator utilização de tecnologias em relação a mão de obra, o $26^{\circ}$ no fator que corresponde ao uso de tecnologia com relação à área explorada com valor negativo, o que se justifica por ser um estado extenso e que possibilita expansão de área frequente, ou seja, a relação entre o uso de tecnologias e a expansão de áreas ainda é falha, devido aos níveis tecnológicos não serem uniformes e nem alcançarem todas os municípios. E, no fator logística o estado também teve resultado negativo, sendo o $25^{\circ}$ colocado e, apesar de não ser explicado pelos autores do trabalho, este resultado pode ser justificado pela infraestrutura logística limitada, sendo na maioria rodovias em condições ruins, o que dificulta o acesso aos portos de Santos, Paranaguá e Santarém, principais rotas de escoação da produção do estado (OLIVEIRA, 2014).

No caso específico da modernização agropecuária no estado de Mato Grosso, destacam-se os trabalhos de Pereira e Mendes (2002) e Pereira et al. (2006), sendo que o primeiro fez uma abordagem utilizando indicadores de produção, crescimento da produção e número de tratores com dados de 1980, 1996 e 2000 e, o segundo, avaliou os efeitos sociais da agricultura no Mato Grosso, ambos utilizando números índices como método. Ambos os trabalhos concluem que a modernização vem evoluindo no estado e ocorre pelas inovações mecânicas, físico-químicas e biológicas, por novos processos organizativos e gerenciais, porém vem acompanhada de desigualdade na distribuição de renda e aumento da pobreza.

Esse fato ocorre por causa do aumento no tamanho da área para viabilizar a escala de produção, que é feita com a incorporação de áreas menores que se tornam inviáveis neste novo contexto tecnológico de alta substituição de mão de obra por máquinas e equipamentos de precisão. Cuadra (1994) afirma que, na América Latina, a mão de obra agrícola geralmente segue uma tendência crescente, porém a inovação tecnológica que deveria poupar o recurso terra, poupa trabalho devido ao impacto no custo de produção. Conforme Locatel (2012), a incorporação crescente da técnica na produção agrícola, a territorialização da "modernização da agricultura" brasileira, não foi um elemento homogeneizador das condições técnicas, econômicas e sociais. Fornazier e Vieira Filho (2012) afirmam que no Brasil é forte a heterogeneidade e a concentração produtiva, pois os agricultores têm acesso diferenciado em relação a tecnologia e aos novos conhecimentos. Portanto, considerar a heterogeneidade quanto ao tamanho das explorações e a ampla 
variabilidade dos preços dos fatores para cada tipo de exploração, é importante para o desenvolvimento de países como o Brasil.

Este trabalho se assemelha aos mencionados que se referem a nível nacional ou a estados como Minas Gerias e Rio Grande do Sul, por utilizar-se da análise fatorial como metodologia na busca de fatores que definam a modernização do estado e do cálculo de nível de modernização por meio de um índice, neste caso chamado IMAM (Índice de Modernização Agrícola dos Municípios). Se diferencia por considerar variáveis relacionadas à novas práticas na agricultura (de menor impacto ambiental), considerando variáveis relacionadas a uma agricultura mais sustentável, tais como plantio direto, cultivo mínimo e agricultura orgânica, além de incorporar a educação para mensurar o efeito da qualificação sobre a modernização do estado. $E$ ainda, quanto aos trabalhos já realizados sobre modernização de Mato Grosso, o diferencial também está em utilizar dados do Censo Agropecuário mais recente, 2006 e, por utilizar Análise Fatorial como método, sendo então inédito para o estado.

Considerando que a colonização de Mato Grosso iniciou de fato na década de 1980, pós Revolução Verde (1960), o estado já começa sua produção agrícola com tecnologias mais avançadas que os demais, por isso atualmente pode-se considerar como um dos estados mais avançados em tecnologia agrícola. Como citado por Vieira Júnior, Figueiredo e Reis. (2014), em 2010 o estado liderou a compra de máquinas e equipamentos direcionados para agricultura (colhedeiras, tratores, pulverizadores), o que pode-se considerar reflexo dos investimentos em técnicas avançadas de produção ocorridas nas últimas três décadas.

Segundo Fuglie (2012) apud Buainain et al. (2014) o sucesso da agricultura brasileira depende de avanços tecnológicos na produção. O uso de transgênicos e de tecnologias de precisão e automação são técnicas novas que impõe desafios, seja com plantas mais eficientes ou melhoria do sistema produtivo. Porém, nem só a tecnologia auxilia na modernização, envolve-se ainda fatores econômicos, sociais e institucionais.

O uso intensivo de máquinas e insumos químicos, associados à contribuição de pesquisas na área agrícola, elevou a produtividade das lavouras e contribuiu para posicionar o Brasil como um dos maiores produtores de alimentos do mundo. Todavia, por problemas de sua distribuição e do baixo poder aquisitivo da população, a melhoria na segurança alimentar é limitada, visto que parte da produção não é acessada pelas pessoas, ou seja, o problema da fome não resulta da produção insuficiente, mas, principalmente, pela falta de renda para acessá-la, aspecto que não é o foco de discussão deste artigo (GASQUES; VIEIRA FILHO; NAVARRO, 2010; PEREIRA et al., 2012).

Assim, para avaliar a modernização de Mato Grosso, deve-se considerar além de máquinas e equipamentos, as variáveis com foco na biotecnologia, em técnicas modernas de cultivo e na gestão agropecuária, pois influem diretamente na produção atual do estado. Sabe-se que já não é mais suficiente gerir uma propriedade com base no conhecimento empírico, logo o nível educacional é importante variável a ser incluída. Considera-se, também o uso de adubos e agrotóxicos, devido seu uso constante nas lavouras do estado. Diante disso, o problema de pesquisa está em identificar qual o nível de modernização dos municípios de Mato Grosso com base nesses padrões atuais de modernização. Ou seja, o estado de Mato Grosso se destaca na produção agrícola moderna de altos insumos ao contribuir para gerar divisas com os saldos positivos na balança comercial brasileira. Com efeito, o objetivo do artigo é identificar e analisar os fatores que determinam a modernização da agricultura nos municípios do estado e apresentar sua configuração por meio do Índice de Modernização Agrícola Municipal. 


\section{MATERIAL E MÉTODOS}

Os dados utilizados na estimativa dos fatores e construção dos indicadores de modernização agrícola para os municípios de Mato Grosso foram obtidos do Censo Agropecuário de 2006, disponibilizado pelo Instituto Brasileiro de Geografia e Estatística (IBGE, 2006). A amostra abrangeu os 141 municípios do estado e as variáveis foram padronizadas da seguinte forma: área de lavoura temporária (ALT) inclui apenas a área cultivada de lavouras temporárias, em hectares; pessoal ocupado (PO) - inclui todo o pessoal ocupado no setor agropecuário de Mato Grosso e; número total de estabelecimentos (TE) - representa o número total de estabelecimentos relacionado com cada variável.

As variáveis selecionadas foram: $X_{1}$ - Valor do investimento $(R \$ 1.000 / A L T)$; $\mathrm{X}_{2}$ - quantidade de combustível - gasolina e óleo diesel (1.000 I/ALT); $X_{3}$ - energia elétrica utilizada (externa e gerada no local)/(ALT); $X_{4}$ - educação - pessoas que dirigem os estabelecimentos com mais de 8 anos de estudo/(ALT); $X_{5}$ - pessoal ocupado com produção de lavoura temporária/(ALT); $X_{6}$ - número de tratores existentes nos estabelecimentos agropecuários/(ALT); $X_{7}$ - número de tratores existentes nos estabelecimentos agropecuários/(PO); $\mathrm{X}_{8}$ - número de máquinas e implementos agrícolas existentes nos estabelecimentos agropecuários/(ALT); $X_{9}$ número de máquinas e implementos agrícolas existentes nos estabelecimentos agropecuários /(PO); $\mathrm{X}_{10}$ - estabelecimentos com cultivo convencional (aração mais gradagem) ou gradagem profunda/(TE); $\mathrm{X}_{11}$ - estabelecimentos com cultivo mínimo (só gradagem)/(TE); $\mathrm{X}_{12}$ - estabelecimentos com agricultura orgânica/(TE); $\mathrm{X}_{13}$ estabelecimentos com cultivo na palha/(TE); $X_{14}$ - estabelecimentos que receberam orientação técnica/(TE); $X_{15}$ - estabelecimentos que fazem controle de pragas/(TE); $\mathrm{X}_{16}$ - estabelecimentos com uso de terraços/(TE); $\mathrm{X}_{17}$ - estabelecimentos com uso de força de tração animal/(TE); $\mathrm{X}_{18}$ - estabelecimentos com uso de agrotóxico por aeronave-(variável dummy - VD). A variável $\mathrm{X}_{18}$ é apresentada como variável dummy (VD), sendo atribuído o valor 0 para ausência desta prática nos municípios e 1 para presença.

Nesse conjunto de variáveis, o uso de combustíveis é justificado, apesar de ser um recurso fóssil exaurível e poluidor, por ser extremamente importante para ampliação da produção agrícola devido ao uso intensivo de máquinas e equipamentos e não ter substituto competitivo no mercado. Além disso, as variáveis cultivo mínimo, convencional e na palha foram incluídas na tentativa de captar a modernização com foco em técnicas de produção de menor impacto ambiental.

Devido ao caráter multidimensional considerado no conceito de modernização agrícola foi aplicada, no presente artigo, a Análise Fatorial. O processamento dos dados foi realizado no software Statistical Package of Social Science - SPSS (IBM Statistic 18).

A Análise Fatorial fornece a estrutura das inter-relações (correlações) entre as variáveis estudadas, definindo um conjunto de dimensões latentes comuns que facilitam a compreensão da estrutura da nuvem de dados, chamadas de fatores (HOFFMANN, 1992). Tal análise busca resumir a quantidade de informações em um conjunto reduzido de variáveis, os fatores, capazes de representar os dados (SANTANA, 2007; SANTANA et al., 2014).

$\mathrm{Na}$ Análise Fatorial, cada variável foi definida como uma combinação linear dos fatores comuns que irão explicar a parcela da variância contida em cada variável, mais um desvio que resume a parcela da variância total não explicada por esses fatores. A parcela da variância explicada pelos fatores comuns recebe o nome de comunalidade e pode variar de 0 a 1 , sendo que valores próximos de 0 (zero) 
indicam que os fatores comuns não explicam a variância e valores próximos de 1 (um) indicam que a variância de todas as variáveis é explicada pelos fatores comuns (HAIR et al., 2009).

A forma matricial do modelo de Análise Fatorial conforme Dillon e Goldstein (1984) é:

$$
X=\alpha F+\varepsilon
$$

Em que: $X$, representado por $\left(x_{1}, x_{2}, \ldots, x_{p}\right)$, é o $p$-dimensional vetor transposto das variáveis observáveis; $\alpha$ é a matriz $(\mathrm{p}, \mathrm{q})$ de constantes desconhecidas, denominadas de cargas fatoriais; $F$, indicado como $\left(f_{1}, f_{2}, \ldots, f_{q}\right)$, é o q-dimensional vetor transposto de variáveis não observáveis ou variáveis latentes chamadas de fatores comuns, sendo que $q<p ; \varepsilon$, representado por $\left(e_{1}, e_{2}, \ldots, e_{p}\right)$, é o p-dimensional vetor transposto de variáveis aleatórias ou fatores únicos..

$\mathrm{Na}$ especificação da Análise Fatorial pressupõe-se que os fatores latentes são não correlacionados entre si e com todos os fatores comuns, ou seja, normalmente, $E(\varepsilon)=E(F)=0$ e $\operatorname{Cov}(\varepsilon, F)=0$. Porém, a estrutura inicial utilizada para determinar a matriz de cargas fatoriais pode não fornecer um padrão significativo de cargas das variáveis, por isso não é definitiva (JOHNSON; WICHERN, 1992 apud GAMA et al., 2007).

Para contornar este problema, utilizou-se o método Varimax de rotação ortogonal dos fatores. O objetivo da rotação é redistribuir a variância dos primeiros fatores para os demais e atingir um padrão fatorial mais simples e teoricamente mais significativo (HAIR et al., 2009; SANTANA et al., 2014).

Para verificar a qualidade das correlações entre as variáveis foram utilizados os testes de Kaiser-Meyer-Olkin (KMO), que se baseia no princípio de que, a inversa da matriz de correlação se aproxima da matriz diagonal, para tanto compara as correlações entre as variáveis observáveis e, o teste de Bartlett, o qual avalia a significância geral da matriz de correlação, testando a hipótese nula de que a matriz de correlação é uma matriz identidade. Desta forma, o KMO maior que 0,5 indica adequação da técnica e quanto mais próximo a 1 (um) indica perfeita adequação dos dados para Análise Fatorial (MOORI; ZILBER, 2003; HAIR et al., 2009). A significância do teste de Bartlett a $1 \%$ permite rejeitar a hipótese nula, indicando que a amostra se adequa à análise. A definição das variáveis relacionadas a cada fator foi feita com base na magnitude e significância das cargas fatoriais, assim cada variável pertence ao fator em que sua carga for mais alta (SANTANA et al., 2014).

A partir dos fatores comuns extraídos, foram estimados os escores fatoriais para cada um dos municípios. Tais escores foram utilizados na construção do Índice de Modernização Agrícola Municipal, visando fazer a hierarquização dos municípios de acordo com o grau de modernização.

Com base em Gama et al. (2007), considera-se que o Índice de Modernização Agrícola Municipal é uma combinação linear dos escores fatoriais e a proporção da variância explicada por cada fator em relação à variância comum. É representado matematicamente por:

$$
I_{M A M_{i}}=\sum_{j=1}^{q}\left(\frac{\lambda_{j}}{\sum_{j} \lambda_{j}} F P_{i j}\right),(i=1,2, \ldots, n)
$$


Em que: $\lambda=$ é a variância explicada por cada fator; $\Sigma \lambda=$ é a soma total da variância explicada pelo conjunto de fatores comuns; $F P=$ escore fatorial padronizado.

Para calcular o índice é necessário fazer a padronização do escore fatorial, para se obter valores positivos dos escores originais sendo possível hierarquizar os municípios, dado que os valores do IMAM estão entre zero e um. A fórmula para a padronização dos escores é:

$$
\mathrm{FP}_{\mathrm{i}}=\left(\frac{F_{i}-F_{\min }}{F_{\max }-F_{\min }}\right)
$$

Em que: $F_{i}=$ escore fatorial original; $F_{\min }=$ valor mínimo observado para os escores fatoriais; $F_{\max }=$ valor máximo observado para os escores fatoriais. Assim, valores de IMAM igual ou maior que 0,70 são considerados altos, se referindo à municípios com alto grau de modernização agrícola; valores entre 0,40 e 0,69 considera-se intermediários e; valores abaixo de 0,40 são os municípios que apresentam baixo IMAM (GAMA et al., 2007).

\section{RESULTADOS E DISCUSSÃO}

Os testes $\mathrm{KMO}$ e Bartlett validam a amostra considerada para esta análise fatorial, sendo KMO com valor igual a 0,690, o que indica adequação da amostra e, o teste de Bartlett com valor de 1.449,26, que também indica a significância das correlações ao nível de $1 \%$ de probabilidade, ou seja, a matriz de correlação não é singular.

Os cinco fatores extraídos entre as 18 variáveis explicam juntos $72,42 \%$ da variância total, que é um valor satisfatório. O primeiro fator comum extraído possui maior importância e explica $23,69 \%$ da variância total dos dados, seguido dos demais fatores que explicam 16,03; 12,78; 10,19 e 9,71\%, respectivamente, da variância total.

Todas as comunalidades foram acima de 0,5 (Tabela 1), portanto, aceitáveis para a inclusão das variáveis no modelo estimado, exceto as variáveis $X_{1}$ e $\mathrm{X}_{13}$ que apresentaram comunalidade 0,4 , mas foram mantidas no modelo por serem relevantes para demonstrar a capacidade de investir e inovar na agricultura matogrossense. A matriz de cargas fatoriais do modelo fatorial estimado, de acordo com sua significância, permite associar as variáveis a cada dimensão latente (Tabela 1).

O primeiro fator extraído incluiu as variáveis $X_{1}$ - valor do investimento; $X_{2-}$ quantidade de combustível - gasolina e óleo diesel; $X_{3^{-}}$energia utilizada (externa e gerada no local); $\mathrm{X}_{4}$ - educação - pessoas que dirigem os estabelecimentos com mais de 8 anos de estudo; e $X_{50}$ - pessoal Ocupado com produção de lavoura temporária. As variáveis educação e pessoal ocupado estão relacionadas ao capital humano e às variáveis investimento, combustível e energia, associam-se ao capital físico, que juntas podem representar a dimensão "Fator Produtivo". Observam-se que as cargas fatoriais de todas as variáveis são positivas e significativas, o que indica bom desempenho produtivo na agricultura.

O agrupamento destas variáveis em um único fator deve-se ao fato de que a utilização de máquinas e equipamentos avançados demandam combustíveis e energia e, para serem operados, também exigem mão de obra qualificada. São as mudanças no "como produzir" que tem feito a produtividade agrícola aumentar. E, mesmo a educação não sendo imprescindível para que ocorra inovação, ela é uma 
facilitadora do processo na agricultura, na medida que auxilia no manuseio de produtos químicos e das máquinas e equipamentos, permitindo a leitura de rótulos e de manuais, por exemplo. Segundo Gasques et al. (2010) entre 1970 e 2006 o peso da mão de obra na agricultura reduziu de $51 \%$ para $16 \%$, o uso de tratores aumentou de $7 \%$ para $17,8 \%$ e os custos, que incluem adubos, energia, agrotóxicos aumentaram de $12 \%$ para $34 \%$.

Tabela 1 - Matriz de cargas fatoriais rotacionadas da modernização agrícola dos municípios do estado do Mato Grosso em 2006.

\begin{tabular}{ccccccc}
\hline \multirow{2}{*}{ Variáveis } & \multicolumn{5}{c}{ Fatores } & \multirow{2}{*}{ Comunalidade } \\
\cline { 2 - 6 } & $\mathrm{F} 1$ & $\mathrm{~F} 2$ & $\mathrm{~F} 3$ & $\mathrm{~F} 4$ & $\mathrm{~F} 5$ & \\
\hline X1 & 0,489 & $-0,158$ & 0,075 & $-0,440$ & $-0,028$ & 0,465 \\
X2 & 0,771 & 0,168 & 0,005 & 0,031 & $-0,157$ & 0,648 \\
X3 & 0,930 & $-0,030$ & 0,039 & $-0,007$ & $-0,074$ & 0,874 \\
X4 & 0,891 & 0,195 & $-0,049$ & $-0,092$ & $-0,060$ & 0,847 \\
X5 & 0,893 & $-0,182$ & $-0,033$ & $-0,046$ & $-0,117$ & 0,847 \\
X6 & 0,607 & 0,694 & $-0,044$ & $-0,113$ & $-0,198$ & 0,904 \\
X7 & $-0,104$ & 0,903 & $-0,064$ & 0,049 & 0,185 & 0,868 \\
X8 & 0,572 & 0,710 & $-0,040$ & $-0,128$ & $-0,178$ & 0,881 \\
X9 & $-0,061$ & 0,941 & $-0,059$ & 0,021 & 0,169 & 0,921 \\
X10 & $-0,068$ & 0,046 & 0,848 & 0,024 & 0,073 & 0,732 \\
X11 & 0,157 & $-0,114$ & 0,751 & 0,184 & $-0,027$ & 0,636 \\
X12 & $-0,051$ & $-0,090$ & 0,719 & $-0,049$ & $-0,239$ & 0,587 \\
X13 & $-0,175$ & 0,028 & $-0,092$ & 0,599 & 0,182 & 0,431 \\
X14 & $-0,175$ & 0,001 & 0,500 & 0,613 & 0,093 & 0,665 \\
X15 & 0,276 & $-0,166$ & 0,235 & 0,685 & $-0,085$ & 0,635 \\
X16 & $-0,088$ & 0,088 & 0,264 & 0,388 & 0,657 & 0,667 \\
X17 & 0,097 & $-0,114$ & 0,275 & 0,347 & $-0,747$ & 0,776 \\
X18 & $-0,278$ & 0,042 & $-0,146$ & 0,294 & 0,683 & 0,652 \\
\hline Variância & 23,690 & 16,037 & 12,789 & 10,192 & 9,713 & 72,422 \\
explicada (\%) & & & & & &
\end{tabular}

Fonte: Dados da Pesquisa.

Para Arrow (1962) a taxa de crescimento da eficácia da mão de obra é resultado do acúmulo de experiência na produção das "commodities", chamando então de "learnig by doing". De maneira diferente, Rosenberg (1982) conceitua o "learning by using", que trata não da experiência, mas do processo de utilização da tecnologia pelo usuário final, considerando que o uso permite o aprendizado. Já para Barros (2014) o "learn by doing" já não é suficiente para os avanços na agricultura, cada vez mais se demanda mão de obra qualificada, com domínio tanto de máquinas quanto de técnicas inovadoras.

Entretanto, apesar do avanço tecnológico existente, observa-se que em regiões de fronteira agrícola os agricultores desbravadores ainda iniciam seus trabalhos com a experiência que trazem de outros locais, como foi caso dos sulistas quando colonizaram Mato Grosso na década de 1980, os quais tiveram diversos percalços na produção de arroz e soja até encontrarem as cultivares adaptáveis ao clima e solo do estado. E ainda, os mesmos produtores de Mato Grosso ao avançaram no território paraense ou mesmo em estados como Piauí, Bahia e Tocantins, já na década de 1990, percebem a necessidade de mudanças de práticas somente depois de utilizarem suas práticas habituais e estas não gerarem bons resultados. 
Malmann e Lago (2012), a partir de uma pesquisa com agricultores no sul do país, concluem que os eles vêm incorporando tecnologias modernas, porém essas passam por algumas adaptações para poderem ser utilizadas, como a substituição de pneus de máquinas e equipamentos adaptados para terrenos acidentados e em desnível. Os autores ainda afirmam que "todas as tecnologias, na avaliação dos agricultores, facilitaram a vida no campo, principalmente por diminuir o trabalho manual e, consequentemente, o tempo e esforço despendidos para fazer as atividades" (MALMANN; LAGO, 2012, p. 37).

O segundo fator está representando a interação de quatro variáveis: $X_{6^{-}}$ Número de tratores existentes nos estabelecimentos agropecuários/(ALT), $\mathrm{X}_{7^{-}}$ Número de tratores existentes nos estabelecimentos agropecuários/(PO), $\mathrm{X}_{8^{-}}$ Número de máquinas e implementos agrícolas existentes nos estabelecimentos agropecuários/(ALT) e $\mathrm{X}_{9}$ - Número de máquinas e implementos agrícolas existentes nos estabelecimentos agropecuários/(PO), sendo relacionadas ao uso de máquinas e equipamentos por área e por pessoal ocupado, o que representa a dimensão "Agricultura mecanizada". A partir deste fator, verifica-se que há distinção entre a relação maquinário/mão de obra e a relação maquinário/área cultivada, que indicam respectivamente a relação de uso da tecnologia por pessoal ocupado e por hectare cultivado.

Todas as variáveis apresentaram carga positiva. As cargas mais altas foram para as varáveis $X_{7}$ e $X_{9}$, que expressam a relação entre máquinas e tratores e uso da mão de obra, ou seja, um alto uso de tecnologia para otimizar o uso da mão de obra, o que reafirma a constante redução da mão de obra disponível no campo. As variáveis $X_{6}$ e $X_{8}$ demonstram que a otimização do uso de máquinas e tratores em relação ao uso da terra também é alta em Mato Grosso, ou seja, a expansão da área vem acompanhada de uso cada vez mais intensivo de máquinas e equipamentos.

Este fator vem de encontro a teoria da inovação induzida iniciada por Ruttan (1985) e Hayami e Ruttan (1988), quando mencionam que as tecnologias são desenvolvidas para facilitar a substituição de fatores escassos e mais caros, por outros abundantes e mais baratos. Exemplifica-se com o caso da substituição de mão-de-obra e terra por insumos menos onerosos. Sendo a substituição da mão de obra relacionada a mecanização e, a substituição da terra ligada a tecnologias biológicas e químicas. Com mudanças nos preços relativos, os produtores rurais são levados a procurar alternativas técnicas poupadoras de fatores que são cada vez mais onerosos.

$\mathrm{O}$ fator três, composto pelas variáveis $\mathrm{X}_{10^{-}}$Estabelecimentos com cultivo convencional (aração mais gradagem) ou gradagem profunda/(TE), $\mathrm{X}_{11^{-}}$ Estabelecimentos com cultivo mínimo (só gradagem)/(TE) e $X_{12^{-}}$Estabelecimentos com agricultura orgânica/(TE), agrupou variáveis relacionadas a métodos de produção de menor impacto ambiental, podendo representar a dimensão "Agricultura de Menor Impacto Ambiental". Este fator indica que no estado, utilizam-se técnicas de produção que visam mitigar os impactos das atividades agrícolas sobre o meio ambiente. A maior carga foi para $X_{10}$, que assim como $X_{11}$, indica boa prática no sentido de evitar erosão e auxiliar na incorporação de fertilizantes e no combate de pragas, além de evitar degradação do solo (AGEITEC, 2016).

Assim como estas práticas, a técnica do plantio direto (inclusa no fator quatro), também é uma alternativa de menor degradação do solo e que melhora o potencial genético de produção das culturas, permitindo custos menores e maximização da produtividade de insumos e de mão de obra (HERNANI, 2014). Esta técnica é muito praticada no estado e pode ser incluída entre as práticas de cultivo mínimo. Assim, é importante destacar que este fator captou o efeito positivo destas práticas já no Censo Agropecuário de 2006. 
Possas et al. (1996), utilizam o conceito de áreas-problema para explicar essas trajetórias tecnológicas e a formação de um novo modelo tecnológico na agricultura, ou seja, a partir de um problema detectado, surgem soluções tecnológicas. Assim, no caso do plantio direto, o grande motivador de pesquisas foi a demanda dos agricultores para reduzir a erosão que refletia na rentabilidade. Para Salles Filho (1993), as áreas-problema no processo de produção agrícola transformam-se em áreas de interesse com o passar do tempo.

$\mathrm{O}$ quarto fator incluiu as variáveis $\mathrm{X}_{13}-$ Estabelecimentos com cultivo na palha/(TE), $X_{14^{-}}$Estabelecimentos que receberam orientação técnica/(TE) e $X_{15^{-}}$ Estabelecimentos que fazem controle de pragas/(TE). Este fator representa a dimensão "Agricultura Moderna", dado que demonstra a existência de acompanhamento técnico, que contribui para a utilização de técnicas agrícolas avançadas, como o plantio direto, indicando que as propriedades agrícolas têm características de produção de mais de um cultivo anual (geralmente soja e milho), além de terem efetivo controle de pragas.

Dentre as vantagens do plantio direto ou plantio na palha está a redução da erosão, sendo comprovado por estudos que com uso desta técnica de cultivo as perdas de solo e água reduzem em torno de $75 \%$ e $22 \%$, respectivamente, variando a cada clima, tipo de solo, cultura e relevo. (BORGES FILHO, 2001). E, conforme dados apresentados por Vieira Júnior, Figueiredo e Reis (2014), a utilização do sistema de plantio direto e de outras técnicas de manejo de produção, ocupavam em 2010 cerca de 500 mil hectares, em 41 municípios de Mato Grosso.

É importante destacar também que o plantio direto é uma das práticas incentivadas pelo Plano ABC (Agricultura de Baixo Carbono), a partir do qual objetiva-se reduzir a emissão de gases de efeito estufa. Por ser um sistema mais complexo, o plantio direto exige mais do agricultor, pois é preciso ter maior qualificação e ter um bom gerenciamento e treinamento da mão de obra, para ter domínio de todas as fases do sistema. Como exemplos de sucesso deste sistema tem-se o cultivo de soja/milho safrinha mais pastagem e, o sistema integrado soja/milho/algodão, o qual associa a rotação de espécies envolvendo culturas de alto valor econômico (AGEITEC, 2016).

Conforme resultados da Fatorial, dos 91 municípios que apresentaram escore fatorial, $42,8 \%$ tiveram influência positiva neste fator, o que demonstra que os produtores do estado estão incorporando estas novas tecnologias e modernizando suas lavouras, porém ainda há municípios em que estas práticas não são adotadas de forma tão intensa, sendo estes a maioria $(57,2 \%)$, o que também pode ocorrer devido ao caráter extensivo da produção agrícola matogrossense.

$O$ quinto fator incluiu as variáveis $X_{16^{-}}$Estabelecimentos com uso de terraços/(TE), $\mathrm{X}_{17^{-}}$Estabelecimentos com uso de força de tração animal/(TE) e $\mathrm{X}_{18^{-}}$ Estabelecimentos com uso de agrotóxico por aeronave - (VD). Diante disso, essa dimensão pode ser nomeada como "Agricultura de altos insumos". A variável $X_{17}$ apresentou carga fatorial negativa de $-0,747$, ou seja, a medida que se inserem altas tecnologias no meio agrícola, as práticas tidas como rudimentares, atreladas à pequena agricultura, não se complementam com a agricultura comercial de alta tecnologia. Outro aspecto que pode ter influência sobre esta variável é o êxodo rural, que vem aumentando nas últimas décadas, fazendo com que pequenas propriedades, a mercê da pobreza, sejam incorporadas por grandes latifúndios, cujo foco é a agricultura de altos insumos e tecnologia. A policultura passa a dar lugar a monocultura de milho e soja, por exemplo e, o boi é substituído pelo trator (ZAMBERLAM; BAIOCCHI; FLORÃO, 1989).

As demais variáveis do fator apresentaram cargas positivas e com valores de mesma magnitude, o que reafirma o uso de técnicas modernas na agricultura de 
Mato Grosso, sendo os terraços uma alternativa que evita erosão e perdas de sementes, devido a contenção da água e, o uso da aeronave (geralmente em grandes áreas) evita maior degradação do solo e perdas de produção, por evitar a entrada de tratores para aplicação dos agrotóxicos.

A partir dos fatores mencionados acima, observa-se que Mato Grosso vem se destacando na incorporação destas variáveis modernas na produção agrícola, sendo importante demonstrar o comportamento de cada município diante de tais fatores.

\section{1. ÍNDICE DE MODERNIZAÇÃO AGRÍCOLA MUNICIPAL (IMAM)}

O IMAM permitiu posicionar os municípios com respeito às fragilidades e pontos fortes quanto ao grau de modernização da agricultura de Mato Grosso. Dos 141 municípios matogrossenses, 50 não apresentaram os escores fatoriais devido à falta de informações na base de dados do Censo Agropecuário, sendo então excluídos da análise do IMAM. A média do IMAM dos 91 municípios foi de 0,2664 (Tabela 2), ou seja, o nível médio de modernização agrícola dos municípios matogrossenses em 2006 foi enquadrado como baixo.

Do total de municípios, 34,07\% registraram um IMAM acima da média, indicando que estes municípios são os que incorporam mais as tecnologias disponíveis para a agricultura do que os demais, sendo a maioria pertencente à região Norte do estado, considerada como principal polo de produção agrícola (IBGE, 2016).

De acordo com os níveis de modernização descritos na metodologia, apesar de terem fatores indicando agricultura moderna e de altos insumos, nenhum município atingiu nível alto de modernização agrícola (acima de 0,7). Pode-se entender este resultado em função da utilização das técnicas que ainda não alcançavam uma distribuição homogênea e generalizada entre os municípios em 2006. Além disso, os municípios com alto padrão de modernização não predominarem sobre os demais, conforme indicado pelos fatores, pode ter ocorrido pelo fato de cada município incorporar apenas alguns dos fatores modernos encontrados na pesquisa, pois na maioria dos casos dos cinco fatores pelo menos um teve carga negativa em cada município, o que puxa o índice para baixo, como é o caso de Sorriso - MT, que está entre os maiores produtores de soja no Brasil e apresentou carga negativa no Fator Produtivo e no Fator Agricultura de Menor Impactos Ambiental. Outro ponto se deve ao caráter extensivo de cultivo predominante no estado nas últimas décadas e que vem sendo modificado nos anos mais recentes, principalmente após a aprovação do Novo Código Florestal em 2012.

A maioria dos municípios, 94,51\% , foram classificados com baixo nível de modernização e, 5,49\% tiveram nível de modernização intermediário. Um fato que contribui para isto foi a inclusão de novas variáveis relacionadas à qualificação de mão de obra e técnicas agrícolas de menor impacto ambiental, que são cada vez mais importantes devido à sustentabilidade agrícola e, no entanto, apresentam baixa distribuição entre os municípios. 
Tabela 2 - Valores dos escores fatoriais originais e o Índice de Modernização Agrícola Municipal (IMAM). ${ }^{1}$

\begin{tabular}{|c|c|c|c|c|c|c|}
\hline Município & F1 & F2 & F3 & F4 & F5 & IMAM \\
\hline Carlinda & 5,7975 & $-1,1660$ & $-0,1780$ & 1,5387 & $-0,2105$ & 0,5379 \\
\hline Rondonópolis & 0,6039 & $-0,3244$ & 3,2120 & 2,0110 & 1,9774 & 0,5004 \\
\hline Porto Estrela & 1,6985 & 7,2928 & 0,0791 & $-0,9752$ & $-1,1070$ & 0,4815 \\
\hline Cuiabá & 3,9007 & $-0,1963$ & $-0,1376$ & $-0,0712$ & 1,2343 & 0,4706 \\
\hline Pontes e Lacerda & 2,7614 & 0,5237 & 0,3349 & 0,4434 & $-1,1020$ & 0,4072 \\
\hline Itiquira & $-0,0877$ & 0,6726 & 1,0363 & 0,5239 & 2,8571 & 0,3983 \\
\hline Glória D'Oeste & 2,7994 & 1,3489 & $-0,5347$ & $-0,9088$ & $-0,2945$ & 0,3854 \\
\hline Campo Verde & $-0,0877$ & 0,2566 & 0,9634 & 1,0062 & 2,2221 & 0,3811 \\
\hline Sorriso & $-0,2029$ & 0,6319 & $-0,2317$ & 3,4617 & 1,2517 & 0,3738 \\
\hline Água Boa & $-0,1337$ & $-0,1254$ & 1,5409 & 1,2513 & 0,6047 & 0,3575 \\
\hline Cáceres & $-0,2794$ & 0,1193 & 3,1473 & $-0,0916$ & $-1,4189$ & 0,3364 \\
\hline Várzea Grande & 2,0101 & $-1,6621$ & 1,0316 & $-2,6425$ & 1,5432 & 0,3357 \\
\hline Chapada dos Guimarães & 0,1795 & $-0,3365$ & 1,1090 & 0,3943 & 0,6221 & 0,3319 \\
\hline Campo Novo do Parecis & $-0,3207$ & 0,9280 & $-0,3611$ & 0,7650 & 2,0949 & 0,3288 \\
\hline Tangará da Serra & $-0,1491$ & $-0,0283$ & 1,1303 & 0,9551 & 0,0893 & 0,3247 \\
\hline Rosário Oeste & $-0,5038$ & 0,0434 & 3,3782 & $-1,3117$ & $-0,6096$ & 0,3238 \\
\hline Santo Antônio do Leverger & $-0,2361$ & $-0,4168$ & 1,2454 & 0,8445 & 0,4118 & 0,3204 \\
\hline Terra Nova do Norte & $-0,4242$ & $-0,3904$ & 3,4531 & $-0,9749$ & $-0,9186$ & 0,3200 \\
\hline Nova Xavantina & $-0,1927$ & 0,1656 & 0,4374 & 1,3943 & 0,3114 & 0,3172 \\
\hline São Félix do Araguaia & $-1,0613$ & 2,4224 & 0,6251 & 1,0112 & $-0,2130$ & 0,3171 \\
\hline Canarana & $-0,5805$ & 0,5494 & 1,1982 & $-0,3361$ & 1,0295 & 0,3138 \\
\hline Diamantino & $-0,5227$ & 0,0911 & 1,3649 & $-0,1576$ & 0,5227 & 0,3033 \\
\hline Nova Mutum & $-0,2869$ & 0,1701 & $-0,7193$ & 1,9121 & 1,0970 & 0,3008 \\
\hline Juara & 0,4830 & 0,0624 & 0,2160 & 0,3014 & $-0,4044$ & 0,2962 \\
\hline Barra do Bugres & $-0,5572$ & 1,0408 & 0,2452 & $-0,0930$ & 0,8285 & 0,2925 \\
\hline Sinop & $-0,0454$ & $-0,4079$ & $-0,4334$ & 1,4833 & 0,7009 & 0,2892 \\
\hline Guiratinga & $-0,3444$ & 0,7390 & $-0,2433$ & 1,0620 & 0,2132 & 0,2889 \\
\hline Nova Santa Helena & 1,1815 & $-0,6283$ & $-0,9648$ & 0,4643 & 0,2013 & 0,2870 \\
\hline Alto Araguaia & $-0,1883$ & $-0,1540$ & $-0,1007$ & 0,4866 & 0,9275 & 0,2835 \\
\hline Nova Ubiratã & $-0,3511$ & $-0,5343$ & 0,5158 & 0,3892 & 0,7133 & 0,2818 \\
\hline Juína & 0,4068 & $-0,3502$ & 0,8568 & 1,3090 & $-2,7062$ & 0,2739 \\
\hline Máximo $^{2}$ & 5,7975 & 7,2928 & 3,4531 & 3,4617 & 2,8571 & \\
\hline Mínimo $^{2}$ & $-1,0613$ & $-1,6621$ & $-1,3095$ & $-2,6425$ & $-2,7062$ & \\
\hline IMAM MÉDIO & & & & & & 0,2664 \\
\hline
\end{tabular}

Fonte: Dados da pesquisa.

1. Estão demonstrados apenas os municípios com IMAM acima da média;

${ }^{2-}$ Valores se referem ao máximo e mínimo entre todos os municípios que tiveram escore.

Os municípios com maior IMAM foram Carlinda e Rondonópolis, com indicador de 0,5379 e 0,5004 respectivamente, sendo considerados intermediários em termos de modernização na agricultura. O município de Carlinda apresenta dois escores fatoriais positivos, sendo associados às dimensões fator produtivo (maior carga) e agricultura moderna e, três escores fatoriais negativos, nos fatores relacionados à mecanização, menor impacto ambiental e avanço tecnológico. Já o município de Rondonópolis, apresentou escore negativo apenas no fator de mecanização e teve maior carga positiva no fator agricultura de menor impacto ambiental. E ainda, também com nível intermediário, mas com carga em torno de 0,4, tem-se os municípios de Porto Estrela, Cuiabá e Pontes e Lacerda.

Os cinco municípios de maior IMAM juntos em 2006 tinham 462 estabelecimentos com lavoura temporária, sendo $2,93 \%$ do total de estabelecimentos do estado (15.785). O município com maior número de estabelecimentos foi Rondonópolis, sendo 234 (IBGE, 2006). 
Dos demais municípios que apresentaram os escores, pode-se concluir que os fatores que se apresentam com maior deficiência são respectivamente, o Fator Produtivo e o Fator de Agricultura Mecanizada. Observa-se que tais municípios podem estar com dificuldades quanto à adoção de técnicas agrícolas menos agressivas ao meio ambiente, seja por falta de conhecimento ou de recursos financeiros. O fator que se apresentou mais eficiente foi o Fator de Agricultura de Altos Insumos, indicando que está havendo incorporação de inovação tecnológica no estado, o que vem de encontro com a problemática levantada, indicando que de fato o nível de modernização de Mato Grosso é influenciado pelos novos padrões tecnológicos.

Para o Fator Produtivo considerando os 91 municípios, foram 71 com escores negativos e $20 \mathrm{com}$ escores positivos, sendo o maior escore do município de Carlinda, que em 2006 teve como destaque o cultivo de milho, mas em pequena escala, produzindo 2.400 toneladas (IBGE, 2016). Para o Fator Agricultura Mecanizada, foram 60 escores negativos contra 31 positivos, sendo o maior no município de Porto Estrela, no qual o cultivo de maior destaque foi a soja, com 3.290 toneladas. Ambos os municípios mencionados são pequenos e tiveram produção inexpressiva no cultivo de soja em 2006, cerca de 107 milhões de toneladas, incluindo soja, milho, arroz e algodão. Logo, o fato de terem apresentado os escores mais altos nestes fatores pode ter ocorrido por terem o pouco investimento focado em altas tecnologias na agricultura local.

O Fator Agricultura de Menor Impacto Ambiental teve 58 escores negativos e 33 escores positivos, sendo o maior para o município de Terra Nova do Norte, o que pode ser justificado por ser um município com foco na agricultura familiar e com a presença forte de uma cooperativa, o que remete ao uso menos intensivo de agroquímicos e implementos agrícolas, sendo utilizadas técnicas mais tradicionais de cultivo devido a extensão das áreas. O Fator Agricultura Moderna teve 53 escores negativos e 38 escores positivos, sendo o maior para o município de Sorriso, o que torna-se aceitável na medida que este é o maior produtor do estado e que tem uma agricultura com alto uso de tecnologia, sendo que em 2006 representou 11,48\% da produção de soja de Mato Grosso (IBGE, 2016).

O Fator Agricultura de Altos Insumos teve 40 escores negativos e 51 escores positivos, cujo maior foi no município de Itiquira, que também está entre os que mais produziram soja no estado em 2006 (371.640 toneladas), logo, sendo esta cultura uma das que mais demandam altos insumos isto justifica o destaque deste escore. E ainda, deve-se considerar que é no município de Itiquira que se encontram as primeiras propriedades da família Maggi, uma das pioneiras no cultivo da soja em Mato Grosso, sendo este um município de alta incorporação de tecnologias desde a década de 1980 (IBGE, 2016; SILVA, 2005).

Estes resultados podem servir como guia para os municípios adotarem medidas que auxiliem na melhoria do setor agrícola, atividade principal do estado de Mato Grosso, permitindo atuar no conjunto de variáveis que definem as dimensões de modernização.

\section{CONCLUSÃO}

A partir da Análise Fatorial foram identificados cinco fatores que representam o grau de modernização da agricultura matogrossense focando na incorporação de inovações tecnológicas (produtos e processos) e explicaram $72,42 \%$ da variação total dos dados. A dimensão indicadora da agricultura de menor impacto ambiental explicou apenas $12,79 \%$ e os fatores representativos da agricultura mecanizada e de altos insumos explicaram $25,76 \%$. 
Os níveis de modernização do estado além de diferenciados, indicam que em 2006 ainda predominavam os sistemas que utilizam tecnologias tradicionais e, no outro extremo, os sistemas que incluem variáveis relacionadas ao menor impacto ambiental apresentaram baixa representatividade na agricultura dos municípios de Mato Grosso, o que resultou em um baixo índice de modernização (IMAM). O que é visto na teoria por Usher (1955) e Ruttan (1959), quando mencionam que a tecnologia antiga pode coexistir com a nova, sendo esta transição gradual.

Apesar de ser o maior produtor nacional de soja e milho, representando 20,8\% em 2006 e 26,7\% em 2014 (IBGE, 2016), Mato Grosso ainda não pode ser considerado um estado moderno em termos de variáveis como técnicas e produtos de cultivo modernas, nível educacional e gestão na agricultura, dado que o IMAM da maioria de seus municípios foi baixo.

Dos 31 municípios com IMAM acima da média, 10 estão localizados na mesorregião Norte, dentre eles o município de Carlinda, com maior IMAM do estado. Nesta mesorregião estão situados $47 \%$ do total de estabelecimentos agropecuários de Mato Grosso, sendo responsáveis por $50 \%$ do valor da produção de lavouras temporárias do estado.

A incorporação de variáveis diferenciadas no modelo permitiu identificar que o processo de modernização do estado baseado em variáveis com foco na redução do impacto ao meio ambiente e na melhor gestão não apresentavam significância estatística nos municípios. Ou seja, ainda há muito a se fazer para o estado atingir um alto índice de modernização, principalmente no que se refere à sustentabilidade, desde questões de aplicação da legislação assim como sua fiscalização.

Assim, sendo o governo o indutor da modernização, responsável por incentivar o investimento, deve-se focar nos municípios com baixo IMAM, desde que seja para fortalecer melhorias baseadas nas técnicas de cultivo, na gestão e na mão de obra e não no aumento de área, já que boa parte do estado pertence à Amazônia Legal.

\section{REFERÊNCIAS}

AGÊNCIA EMBRAPA DE INFORMAÇÃO TECNOLÓGICA - AGEITEC. Disponível em: < http://www.agencia.cnptia.embrapa.br/index.html >. Acesso em: 04 mai. 2016.

BALSAN, R. Impactos Decorrentes da Modernização da Agricultura Brasileira. Campo-Território: Revista de Geografia Agrária, v. 1, n. 2, p. 123-151, 2006. Disponível em: <http://www.seer.ufu.br/index.php/campoterritorio/article/view/11787/8293>. Acesso em: 23 mai. 2012.

BARROS, G. S. C. Agricultura e indústria no desenvolvimento brasileiro. In: BUAINAIN, A. M.; ALVES, E.; SILVEIRA, J. M.; NAVARRO, Z. O mundo rural no Brasil do século 21: a formação de um novo padrão agrário e agrícola. Brasília, DF: Embrapa, 2014. 1182p.

BORGES FILHO, E. L. O desenvolvimento do plantio direto no Brasil: a conjunção de interesses entre agricultores, indústria e o estado. 2001. $156 f$. Dissertação (Mestrado em Economia do Meio Ambiente) - Instituto de Economia, Universidade Estadual de Campinas, Campinas, SP, 2001.

BUAINAIN, A. M.; PEDROSO, M. T. M.; VIEIRA JÚNIOR, P. A.; SILVEIRA, R. L. F.; NAVARRO, Z. Quais os riscos mais relevantes nas atividades agropecuárias? In: 
BUAINAIN, A. M.; ALVES, E.; SILVEIRA, J. M.; NAVARRO, Z. O mundo rural no Brasil do século 21: a formação de um novo padrão agrário e agrícola. Brasília, DF: Embrapa, 2014. 1182p.

CAMPOS, S. A. C.; PEREIRA, M. W. G.; TEIXEIRA, E. C. Trajetória de Modernização da Agropecuária Mineira no Período de 1996 a 2006. Economia Aplicada, v. 18, n. 4, p. 717-739, 2014.

COSTA, C. C. M.; REIS, P. R. C.; FERREIA, M. A. M.; MOREIRA, N. C. Modernização Agropecuária e Desempenho Relativo dos estados Brasileiros. Agroalimentaria, Mérida, v. 18, n. 34, p. 43-56, 2012.

CUADRA, F. M. A teoria da inovação induzida: uma crítica. Traduzido do espanhol por José Eugenio Guimarães. Estudos Sociedade e Agricultura, 2, p. 107-112, jun. $1994 . \quad$ Disponível em: < http://bibliotecavirtual.clacso.org.ar/ar/libros/brasil/cpda/estudos/dois/cuadra2.htm>. Acesso em: 31 ago. 2016.

CUNHA, N. R. S.; LIMA, J. E.; GOMES, M. F. M.; BRAGA, M. J. A Intensidade da Exploração Agropecuária como Indicador da Degradação Ambiental na Região dos Cerrados, Brasil. Revista de Economia e Sociologia Rural, v. 46, n. 2, p. 291-323, $2008 . \quad$ Disponível em: <http://ageconsearch.umn.edu/bitstream/61241/2/v46n2a02.pdf>. Acesso em: 23 mai. 2015.

DILLON, W. R.; GOLDSTEIN, M. Multivariate analysis-methods and applications. Wiley, New York: 1984, 587p.

EHLERS, E. M. O que se entende por agricultura sustentável? 1994. $165 \mathrm{f}$. Dissertação (Mestrado em Ciência Ambiental) - Programa de Pós-Graduação FEA/USP, São Paulo,1994. Disponível em: < http://www.teses.usp.br/teses/disponiveis/90/90131/tde-25112011-091132/ptbr.php>. Acesso em: 10 ago. 2016.

FERO. A. O Setor de Máquinas Agrícolas no Brasil: evolução nos últimos anos e perspectivas. Revista Eletrônica Céleres, 2014. Disponível em: $<$ http://www.celeres.com.br/o-setor-de-maquinas-agricolas-no-brasil-evolucao-nosultimos-anos-e-perspectivas/\#>. Acesso em: 15 jun. 2016.

FERREIRA JUNIOR, S.; BAPTISTA, A. J. M. S.; LIMA, J. E. A Modernização Agropecuária nas Microrregiões do estado de Minas Gerais. Revista de Economia e Sociologia Rural, v. 42, n. 1, p. 73-89, 2004. Disponível em: <http://www.scielo.br/pdf/resr/v42n1/20923.pdf>. Acesso em: 21 mai. 2015.

FORNAZIER, A.; VIEIRA FILHO, J. E. R. Heterogeneidade estrutural no setor agropecuário brasileiro: evidências a partir do Censo Agropecuário de 2006. Texto para Discussão, n. 1.708. Brasília: Ipea, 2012.

FUGLIE, K. O. Productivity growth and technology capital in the global agricultural economy. In: FUGLIE, K. O.; WANG, S. L.; BALL, V. E. (Ed.). Productivity growth in agriculture: an international perspective. Oxforshire: CABI International, 2012. p. 365-368. 
GAMA, Z. J. C.; SANTANA, A. C.; MENDES, F. A. T.; KHAN, A. S. Índice de desempenho competitivo das empresas de móveis da região metropolitana de Belém. Revista de Economia e Agronegócio, v. 5, n. 1, p. 127-160, 2007. Disponível em: <http://www.repositorio.ufc.br:8080/ri/bitstream/123456789/1305/1/2007_art_zjcgam a.pdf>. Acesso em: 21 mai. 2015.

GASQUES, J. G.; BASTOS, E. T.; BACCHI, M. R. P.; VALDES, C. Produtividade total dos fatores e transformações da agricultura brasileira: análises dos dados dos censos agropecuários. In: GASQUES, J. G.; VIEIRA FILHO, J. E. R.; NAVARRO, Z. (Org.). A agricultura brasileira: desempenho, desafios e perspectivas. Brasília, DF: Ipea, 2010. p. 19-44.

GASQUES, J. G.; VIEIRA FILHO, J. E. R.; NAVARRO, Z. Agricultura brasileira: desempenho, desafios e perspectivas. Brasília: Ipea, 2010. 298 p. Disponível em: <http://www.ipea.gov.br/sites/000/2/livros/2010/Livro_agriculturabrasileira.pdf >. Acesso em: 10 jun. 2016.

HAIR, J. F; BLACK, W. C.; BABIN, B. J.; ANDERSON, R. E.; TATHAM, R. L. Análise multivariada de dados. 6ed. Porto Alegre: Bookman, 2009. 688p.

HAYAMI, Y.; RUTTAN, V. Desenvolvimento agrícola: teoria e experiências internacionais. Brasília, DF: Embrapa, 1988.

HERNANI, L. C. (Ed.). Sistema plantio direto. Brasília, DF: Embrapa, 2014. Disponível em: <http://www.agencia.cnptia.embrapa.br/gestor/sistema_plantio_direto/ arvore/CONT000fh2b6ju702wyiv80rn0etnxng2vnj.html>. Acesso em: 01 mai. 2016.

HOFFMANN, R. A dinâmica da modernização da agricultura em 157 microrregiões homogêneas do Brasil. Revista de Economia e Sociologia Rural, v. 30, n. 4, p. 271-290, 1992.
INSTITUTO BRASILEIRO DE GEOGRAFIA E ESTATÍSTICA - IBGE. Censo agropecuário do ano de 2006. Disponível em: <http://www.ibge.gov.br/home/estatistica/economia/agropecuaria/censoagro/default.s htm.>. Acesso em: 15 mai. 2012.

INSTITUTO BRASILEIRO DE GEOGRAFIA E ESTATÍSTICA - IBGE. Sistema IBGE de Recuperação Automática. Pesquisa agrícola municipal. Disponível em: < http://www.sidra.ibge.gov.br/bda/pesquisas/pam/default.asp?0=29\&i=P>. Acesso em: 04 mai. 2016.

INSTITUTO DE PESQUISA ECONÔMICA APLICADA - IPEA. Brasil em desenvolvimento 2010: estado, planejamento e políticas públicas. Brasília, DF: 2010. Disponível em: <http://www.ipea.gov.br/bd/pdf/Livro_BD_vol2.pdf. Acesso em 02/2016 $\geq$. Acesso em: 17 jun. 2015.

JOHNSON, R. A.; WICHERN, D. W. Applied multivariate statistical analysis. 3ed Englewood Cliffs, New Jersey: Prentice Hall, 1992. 
LOCATEL, C. Tecnificação dos territórios rurais no Brasil: políticas públicas e pobreza. Scripta Nova: Revista Electrónica de Geografía y Ciencias Sociales, v. XVI, n. 418 (66) , Barcelona: Universidad de Barcelona, 1 de nov. 2012. Disponível em: <http://www.ub.es/geocrit/sn/sn-418/sn-418-66.htm>. Acesso em: 29 ago. 2016.

MALMANN, M. S.; LAGO, I. C. Os Agricultores e a "Modernidade": Uma Análise da Relação entre Cultura e Tecnologia no Meio Rural de Cerro Largo / RS. Extensão Rural, Santa Maria v. 19, n. 1, Jan./Jun. $2012 . \quad$ Disponível em:<https://periodicos.ufsm.br/extensaorural/article/view/6538>. Acesso em: 19 mar. 2017.

MAZZOLENI, E. M.; OLIVEIRA, L. G. Inovação Tecnológica na Agricultura Orgânica: estudo de caso da certificação do processamento pós-colheita. Revista de Economia e Sociologia Rural, v. 48, n. 3, Brasília, Jul./Set. 2010. Disponível: $<$ http://www.scielo.br/scielo.php?script=sci_arttext\&pid=S0103-

20032010000300004>. Acesso em: 14 abr. 2015.

MOORI, R. G.; ZILBER, M. A. Um Estudo da Cadeia de Valores com a Utilização da Análise Fatorial. Revista de Administração Contemporânea, v. 7, n. 3, 2003. Disponível em: <http://www.anpad.org.br/rac-page-normasframe.html>. Acesso em: 24 abr. 2012.

NUNES, S. P. o desenvolvimento da agricultura brasileira e mundial e a ideia de desenvolvimento rural. Departamento de Estudos Socioeconômicos RuraisDESER, 2007. (Boletim Eletrônico- Conjuntura Agrícola, n.157). Disponível em: $<$ http://www.deser.org.br/documentos/doc/DesenvolvimentoRural.pdf>. Acesso em: 05 set. 2015.

PEREIRA, B. D.; MARTINS, V. F.; MENDES, C. M.; FARIA, A. M. M.; SILVA, G. R. Reflexões Sociais sobre Efeitos da Modernização da Agricultura de Mato Grosso. Revista de Estudos Sociais, ano 8, n. 1 e 2, p. 33-48, 2006.

PEREIRA, B. D.; MENDES, C. M. A Modernização da Agricultura de Mato Grosso. Revista de Estudos Sociais, n. 7, p. 61-76, 2002.

PEREIRA, P. A. A.; MARTHA JUNIOR, G. B.; SANTANA, C. A. M.; ALVES, E. The development of Brazilian agriculture, future technological challenges and opportunities. In: MARTHA JUNIOR, G. B.; FERREIRA FILHO, J. B. de (Ed.) Brazilian agriculture development and changes. Brasília, DF: Embrapa, p. 13-42, 2012.

PESSÔA. V. L. S; MATOS, P. F. A Modernização da Agricultura no Brasil e Novos Usos do Território. Revista Geo UERJ, v. 2, n. 22, Universidade Estadual do Rio de Janeiro. Rio de Janeiro/RJ: 2013. Disponível em:<http://www.epublicacoes.uerj.br/index.php/geouerj>. Acesso em: 10 set. 2015.

PINTO, N. G. M.; CORONEL, D. A. Modernização Agrícola no Rio Grande do Sul: Um Estudo nos Municípios e Mesorregiões. Revista Paranaense de Desenvolvimento, v. 36, n. 128, 2015. Disponível em: http://www.ipardes.pr.gov.br/ojs/index.php/revistaparanaense/article/view/712>. Acesso em: 02 fev. 2016. 
POSSAS M.; SALLES FILHO, S.; SILVEIRA, J. M. An evolutionary approach to technological innovation in agriculture: some preliminary remarks. Research Policy, n. 25, p. 933-945, 1996.

RAMMINGER, R.; GRASEL, D.; ZAVALA, A. A. Z. Agricultura e Indicadores de Renda no estado de Mato Grosso (1980-2005). Informe GEPEC. Universidade Estadual do Oeste do Paraná, v. 12, n. 2, p. 56-72, 2008. Disponível em: <http://erevista.unioeste.br/index.php/gepec/article/view/1757>. Acesso em: 10 dez. 2012.

RUTTAN, V. La teoría de la innovación inducida del cambio técnico en el agro de los países desarrolhados, Cambio técnico en el agro latinoamericano. San José, Costa Rica, Piñeiro y Trigo, IICA, 1985.

RUTTAN, V. Usher and Schumpeter on invention, innovation and technological change. Quarterly Journal of Economics, p. 596-606, nov. 1959.

SALLES FILHO, S. L. M. A. Dinâmica tecnológica da agricultura: perspectivas da biotecnologia. 1993. 248f. Tese (Doutorado em Economia) - Instituto de Economia, Universidade Estadual de Campinas: Campinas, 1993. Disponível em: < http://www.bibliotecadigital.unicamp.br/document/?code=vtls000061988>. Acesso em: 10 jul. 2016.

SANTANA, A. C. Índice de desempenho competitivo das empresas de polpa de frutas do estado do Pará. Revista de Economia e Sociologia Rural (Impresso), v. 45, p. 749-775, 2007.

SANTANA, A. C.; SANTANA, Á. L.; SANTANA, ÁDINA L.; COSTA, N. L.; NOGUEIRA, A. K. M. Planejamento Estratégico de uma Universidade Federal da Amazônia: aplicação da Análise Fatorial. Revista de Estudos Sociais (UFMT), v. 32, p. 183-204, 2014.

SANTANA, A. C. A dinâmica do complexo agroindustrial e o crescimento econômico no Brasil. 1994. 302 f. Tese (Doutorado em Economia Rural) Universidade Federal de Viçosa, Viçosa: UFV, 1994.

SEPULCRI, O.; PAULA, N. A evolução da agricultura e seus reflexos na EMATER. Programa de Pós-Graduação em Desenvolvimento Econômico, Universidade Federal do Paraná, Curitiba/PR: 2005. Disponível em: $<$ http://www.emater.pr.gov.br/arquivos/File/Biblioteca_Virtual/Premio_Extensao_Rura I/2_Premio_ER/03_Evol_Agri_refl_Emater.pdf>. Acesso em: 10 mar. 2016.

SILVA, C.A.F. A Transnacionalização do Grupo André Maggi a partir do Cerrado Mato-Grossense. Revista geo-paisagem (on line), ano 4, n. 5, jan-jun, 2005. Disponível em: < http://www.feth.ggf.br/Revista7.htm>. Acesso em: 15 abr. 2016.

SOUZA, P. M.; LIMA, J. E. Intensidade e Dinâmica da Modernização Agrícola no Brasil e nas Unidades da Federação. Revista Brasileira de Economia, v. 57, n. 4, p. 795-821, 2003.

TEIXEIRA, J. C. Modernização da agricultura no Brasil: impactos econômicos, sociais e ambientais. Revista Eletrônica da AGB, v. 2, n. 2, ano 2, p. 21-42, Três Lagoas/MS, set. 2005. 
USHER, A. P. Technical change and capital formation, Capital formation and economic growth. National Bureau of Economic Research, p. 523-550,1955.

VIEIRA JÚNIOR, P. A.; FIGUEIREDO, E. V. C.; REIS, J. C. Alcance e limites da agricultura para o desenvolvimento regional: $O$ caso de Mato Grosso. In: BUAINAIN, A. M.; ALVES, E.; SILVEIRA, J. M.; NAVARRO, Z. O mundo rural no Brasil do século 21: a formação de um novo padrão agrário e agrícola. Brasília, DF: Embrapa, 2014. 1182p.

ZAMBERLAM, J.; BAIOCCHI, M.; FLORÃO, S. R. Cruz Alta: as perspectivas do desenvolvimento - um estudo socioeconômico prospectivo. APROCRUZ: Associação dos Professores de Cruz Alta, Cruz Alta/RS, 1989. 